\title{
The Peculiarities of Acoustical Monitoring of Moving Objects in Shallow Water Areas
}

\author{
Manel Monteiro, Victor Svet, Donald Sandilands \\ International Tank Services (Asia Pacific) PTE Ltd., Singapore City, Singapore \\ Email: vsvetd@mail.ru
}

Received 19 July 2014; revised 18 August 2014; accepted 15 September 2014

Copyright (C) 2014 by authors and Scientific Research Publishing Inc.

This work is licensed under the Creative Commons Attribution International License (CC BY).

http://creativecommons.org/licenses/by/4.0/

\begin{abstract}
The article considers peculiarities of underwater monitoring of moving objects in the shallow water areas, particularly seaports. These areas are characterized by a multitude of factors influencing the efficiency of detection. Nonstationarity conditions of sound propagation and specific interference caused by shipping are the major factors. The various algorithms for the space-time signal processing have been tested and some experimental results are presented. It has been shown that the use of clipped mode in conjunction with the correlation processing of wideband signals and subsequent speckle tracking allow realizing high efficiency of monitoring.
\end{abstract}

\section{Keywords}

Acoustical Underwater Monitoring, Shallow Water Areas, Multi-Ray Propagation, Correlation Processing, Speckle-Tracking

\section{Introduction}

Shallow sea areas, such as sea ports, are characterized by several factors, significantly complicating detection of small moving underwater objects. The main factors are strong multi-ray sound propagation and bottom-surface reverberation. Known solutions or these problems are based on the use of highly directional arrays in two planes and application of complex wideband signals with subsequent correlation processing. The most of short-range sonars use these approaches. There are other factors which significantly influence the detection and tracking-intensive shipping in port areas. Shipping creates two types of noise. First of all moving vessel generates powerful turbulent perturbations (the wakes), which leads to strong amplitude and phase fluctuations in received echo signals. Because of the small depths such disturbances can produce a kind of "phase" screen between the object and the receiving arrays which can completely destroy the wave front of coming echo-signals. The second type of noise is powerful active signals from different echo-sounders installed on ships that operate continuously, 
and noise generated by ship itself. Frequencies of echo sounders may be close to the operating frequency of underwater monitoring systems, and very often they become "blind" for a while. Noise generated by ship is generally in a low frequency range, however, because of its very high intensity and non ideal shape of the input filter frequency response some part of the ship noise may penetrate into signal processing unit. It is known that for specified waveguides the optimal signal processing technique is based on matched filtering processing, MFP [1] [2], and time reversed acoustics, TRA [3]. However, several factors prevent their practical use. MFP is not effective because calculation or measuring of transfer functions of waveguide is impossible to manage them with sufficient accuracy in such conditions. TRA requires the spacing of receiving and transmitting arrays which is often impossible in real port conditions. In this paper we will consider and compare experimental results of different methods of signal processing, namely, standard correlation procedures with a fixed and adaptive threshold in linear and nonlinear modes and speckle tracking signal processing.

\section{Some Theoretical Background}

\subsection{Standard Algorithms of Signal Processing}

Structure of generalized receiving tract of signal processing is presented in Figure 1.

Probe signals are complex wide-band signals with $\Delta F T \gg 1$, where $\Delta F$-frequency band, $T$-duration of probe signal. In accordance with this scheme, we will consider the following algorithms for the space-time signal processing.

- Space processing: 2D beamforming which is a standard procedure based on Fourier processing (compensation of time delays of signals in angle channels with next summation).

- Time processing was realized in two modes:

- linear mode: amplification of input signals in high dynamic range, calculation of correlation function, normalization.

- non-linear mode: amplification of input signals, deep bilateral limitation (clipping), calculation of sign correlation.

- The last procedure is a threshold detection with a fixed threshold ( $\Delta=$ constant) or adaptive threshold $(\Delta=\psi(t, \alpha, \beta))$ and the following decision "yes" or "no".

Formally, the received echo signals in some angle channels $\alpha, \beta$ can be written as

$$
Z_{\alpha, \beta}(t)=\sum s_{i}\left(t, r_{i}\right)+N(t)
$$

where $s_{i}\left(t, r_{i}\right)$ are echo-signals from different distances $r_{i}$ and $N(t)$-additive noise with Gaussian statistics. In a linear mode the output signal after correlation procedure can be written as $R(\alpha, \beta)=\left|Z_{\alpha, \beta}(t) \otimes h(t-\tau)\right| \vee \Delta$, where $h(t-\tau)$-reference signal, $\Delta$-value of threshold. If the value of correlation function is more than the value of threshold, $R(\alpha, \beta) \geq \Delta$, the output signal is considered as detected. In non linear mode the bilateral deep limiter was used with function response

$$
Q(t)=\left\{\begin{array}{l}
U_{0}-\text { if } . Z_{\alpha, \beta}(t)>0 \\
0-\text { if } . Z_{\alpha, \beta}(t)=0 \\
-U_{0}-\text { if } . Z_{\alpha, \beta}(t)<0
\end{array}\right\}
$$

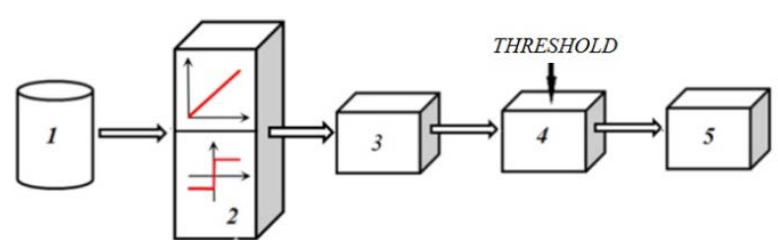

Figure 1. Typical structure of receiving tract of signal processing. 1) Receiving 2D array; 2) Time processing (linear and nonlinear; 3) Space signal processing (2D beamforming); 4) Threshold detection; 5) Output data (display). 
For Gaussian noise the relationship between the correlation function in the linear mode $R_{\text {lin }}(\alpha, \beta)$ and clipped mode $R_{\text {clip }}(\alpha, \beta)$ is given by a well known formula

$$
R_{\text {clip }}(\tau)=\frac{2 a^{2}}{\pi} \arcsin \left\{\frac{R_{\text {lin }}(\tau)}{\sigma^{2}}\right\},
$$

where $a=$ const,$\sigma^{2}$-dispersion of signal. Obviously the dispersion of output signal is equal to $\sigma^{2}=a^{2}$. It is seen that losses because of clipping is about $30 \%$. Despite the great advantages of clipping mode, namely the persistence of a false alarm and substantial simplification of signal processing (minimum dynamic range), its application for active sonar is often questioned by specialists. Their main argument is that a strong object can mask the weak object. This statement is not quite correct, because usually the quantitative relationships between the intensities of the strong and weak signals are not specified in such disputes. Moreover, all such statements are usually considered for stationary noise, which is not typical for the port areas. In the same time, rigorous theoretical analysis shows that clipping allows simultaneous detection of both strong and weak signals, albeit with some loss of noise immunity. One of theoretical results is demonstrated on Figure 2, [4].

It follows from Figure 2, that the losses of output signal/noise ratio for clipping mode are more and less significant only if input signal/noise ratio is more than 2 - 3 (6 - $10 \mathrm{~dB})$. If we will take into account that the input signal/noise ratio for small objects is very small $\left(S_{\text {input }} / N \ll 1\right)$, clipping mode will play the linear mode only for very large input signals. Because in the real world we can meet a variety of cases, it was interesting to consider the use of both modes of processing for the same environments to compare them.

\subsection{Speckle Tracking Methods}

If unknown scattering medium is placed between moving point source and the receiving array the registered field becomes random, and the received signals are characterized by strong amplitude-phase modulation. Angular wave spectrum is greatly extended, and the standard spatial signal processing leads to a large ambiguity of measured coordinates of a point source. Speckle-interferometry methods for measuring the parameters of objects through scattering media are based on the existence of short-time coherence of scattered fields recorded at different moments of time, if during this period the scattering medium was stable [5]-[8]. It is important that the detail information about inhomogeneous medium is not necessary. Thus the information about the source parameters is extracted from correlation characteristics of the scattered fields. First, this principle has been demonstrated in the classical optical work of Burch and Tokarski, [9], and later these methods have resulted in a whole new trend in optics and acoustics, and received different names: "speckle interferometry", "speckle tracking" and "speckle holography". Moreover, it appeared that this idea is applicable to restore acoustical images of extended objects, not just the point objects through scattering medium [8]. Multibeam waveguide is significantly heterogeneous medium and therefore it was interesting whether such speckle methods can be applied to such environments. Theoretical analysis was first published in [3] and below we only state the main results.

First of all let's remind the basic idea of speckle method (Figure 3).

Let inhomogeneous scattering layer is positioned between the moving point sound source and a $2 \mathrm{D}$ receiving array, and we need to measure the trajectory of this source. Properties of the inhomogeneous layer are such that it completely distorts the wave front from the source and the registered fields look like noise images due scattering and so the usual beam forming is senseless. In a moment of time $t=t_{1}$ let's register the intensity of scattered field $I_{1}(x, y)$ on array. In some time $t_{2}=t_{1}+\Delta t$ the point source will slightly change its position and we register the second distribution of intensity $I_{2}(x, y)$. This second image looks like the first image and visually differences between the two images are not detectable. It turns out that if the shift of the point source $\Delta$ satisfies to certain conditions, the two intensity distributions are correlated. Well known Young's fringes are the result of such correlation, Figure 2. Having the result of correlation it is simple to reconstruct the image of point source using Fourier transformation of Q. Note that detail information about parameters of scattering layer to reconstruct the image was not required. As follows from this consideration the speckle principle is very simple, but, of course, its implementation is not possible in all conditions. The main condition is that the speed fluctuation of the scattering layer must be much less than the speed of movement of the point source. 


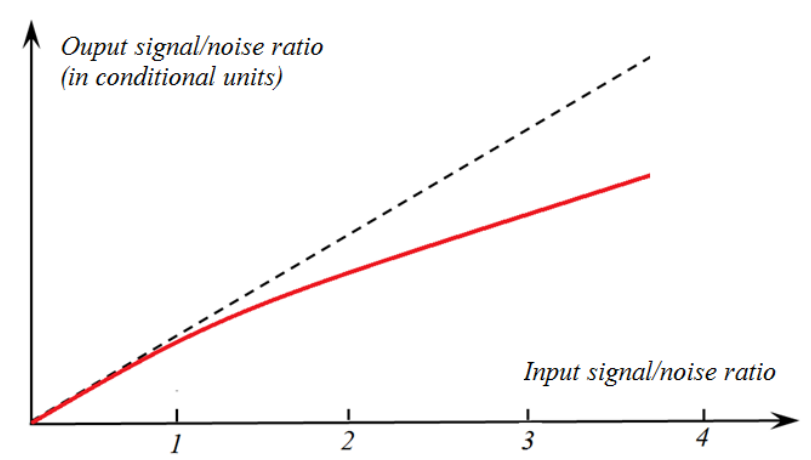

Figure 2. Output signal/noise ratio for linear signal processing system (dashed curve) and clipping system (red curve).

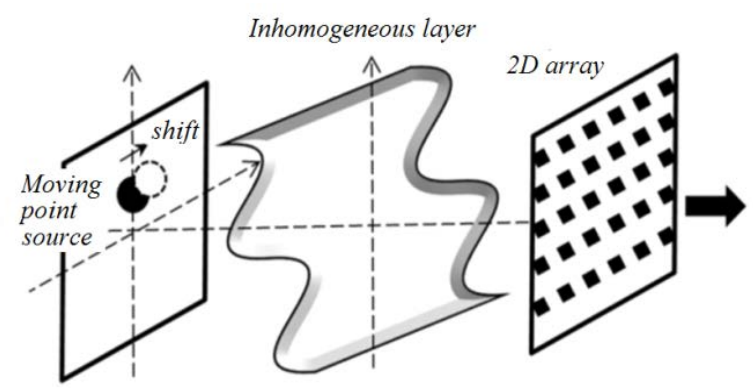

Figure 3. Detection of moving point source through scattering layer.

\subsection{Speckle Tracking in Waveguides}

Acoustic field in the stratified or ideal waveguide can be described as a group of modes or rays. At receiving this field on vertical or horizontal arrays of big waved imensions after standard beam forming we will obtain the output discrete angular spectrum of modes, which leads to ambiguity of direction finding. The basic idea of the speckle method is that under certain assumptions the formed angular spectrum of modes can be regarded as a unique speckle pattern. If at some shift of the echo source, this pattern will not change its structure, speckle interferometry method can be used to measure the trajectory of the source, without resorting to MFP or TRA methods, [6] [7]. Let's place a horizontal antenna in the waveguide at a distance $R$ from echo source. The received field on $i$-th element of array can be written as a sum of running modes:

$$
P\left(x_{i}\right) \approx \sum_{m=1}^{M} A_{m} \exp \left[j \xi_{m}\left(R+\alpha x_{i}\right)\right]
$$

where $A_{m}$-the amplitude of the $m$-th mode, $\xi_{m}=\frac{2 \pi}{\lambda} a_{m}$-the horizontal component of the wave vector, $x_{i}-$ coordinate of the $i$-th receiver, $\alpha$-direction on the sound source. Suppose that $a_{m}=1-b_{m}$, where $b_{m}-$ small increments, but not vertical wave numbers, i.e. $\quad b_{m} \ll 1$. Then (4) can be rewritten as

$$
P\left(x_{i}\right) \approx \exp \left[j k R\left(1+\alpha x_{i}\right)\right] \times \sum_{m=1}^{M} A_{m} \exp \left[-j k b_{m}\left(R+\alpha x_{i}\right)\right]
$$

Equation (5) shows that the phase factor before the sum describes a plane wave incident on the antenna at an angle $\alpha$. If sound source will shift on angle $\alpha^{\prime}$ and distance $R^{\prime}$ factor as the sum in (5) will change its form on $\sum_{m=1}^{M} A_{m} \exp \left[-j k b_{m}\left(R^{\prime}+\alpha^{\prime} x_{i}\right)\right]$. However if to suggest that phase factors in sum (2) keep phase in limits $\pi / 2$ at replacing $\alpha$ and $R$ on $\alpha^{\prime}$ and $R^{\prime}$ accordingly it is possible to consider phase function as constant and similar to persistent speckle pattern. The shifts of the source on depth must be also such that the values of 
$A_{m}$ should not change their signs. Let's propose that source angle shift $\Delta \alpha=\alpha-\alpha^{\prime}=\alpha_{0}$, where $\alpha_{0}=\frac{\lambda}{L}$ angle resolution of horizontal array. If array sampling is $\lambda / 2$ and $N$-is a number of elements, $\alpha_{0}=\frac{2}{N}$. At the assumed condition that allowable phase shift should be less than $\pi / 2$ we can write

$$
\frac{2 \pi}{\lambda} b_{m}\left(R+\alpha x_{i}\right)-\frac{2 \pi}{\lambda}\left[R^{\prime}+\left(\alpha+\alpha_{0}\right) x_{i}\right]=\frac{2 \pi}{\lambda} b_{m}\left(R-R^{\prime}\right)-\frac{2 \pi}{\lambda} b_{m} \alpha_{0} x_{i} \leq \frac{\pi}{2}
$$

Let's $x_{i}=x_{\max }=N \lambda / 2$. Then taking in regard that $\alpha_{0}=\frac{2}{N}$ we can rewrite (6) as

$$
\frac{2 \pi}{\lambda} b_{m}\left(R-R^{\prime}\right)-\frac{2 \pi}{\lambda} b_{m} \lambda=\frac{2 \pi}{\lambda}(\Delta R-\lambda) \leq \frac{\pi}{2}
$$

Therefore the allowable shift on distance will be

$$
\Delta R \leq \frac{\lambda}{4 b_{m}}+\lambda
$$

Because for the most ocean waveguides the values of $b_{m} \approx 10^{-2}-10^{-3}$ the allowable shifts of distances can be tenths and hundreds wavelengths. For example for $\lambda=3 \mathrm{~m}$ and $L=300 \mathrm{~m}$ possible shift of distance can be about $750 \mathrm{~m}$ and phase factor will not change its form that is necessary to implement the method of speckle interferometry [7]. At higher frequencies, this condition becomes more severe, for example for $F=100 \mathrm{kHz}$ the allowable variations in distance between two expositions becomes the order of meters or less. The detail algorithms of signal processing are described in [6].

\section{Experimental Results}

In all experiments we used cylindrical array on frequency $F=100 \mathrm{kHz}$ with horizontal directivity $2^{\circ}$ and vertical directivity about $9^{\circ}$. Horizontal sector of view was changeable from $45^{\circ}$ up to $270^{\circ}$. Probe signals were LFM signals with a bandwidth $27 \mathrm{kHz}$ and different duration. In linear mode TVG was used.

\subsection{Linear Mode and Clipping Mode of Detection}

First of all we want to demonstrate the sonar images in linear mode and clipping mode, Figure 4(a), Figure 4(b). On this Figure 3 one can see many different objects. The right side, marked by red rectangular, is stationary port constructions-breakwater, betony walls and other underwater units. The image in a red oval is unknown echo signal. Red arrows are detected moving small boats. The left low side of images is a zone of strong reverberation, the depth of this zone is no more than 4 meters. There is no principal difference between these two images. Clipping mode is reproducing all details of sonar images in a linear mode and practically with the same contrast and relative intensities except some details of strong reflecting stationary objects. Moreover the tracking of moving boats is more reliable in clipping mode though possibly the law of TVG in linear mode has not been accurate

\subsection{Suppression of Stationary Objects}

Since speckle interferometry methods allocate only nonstationary echo-signals, they can be effectively used to suppress coherent echo signals reflected from stationary objects. Port areas piers, concrete pillars, breakwaters and other underwater structures mounted on the bottom are such objects. Practical implementation of speckleinterferometry methods in this case are similar to the known algorithms of through-periodical subtraction of signals to suppress the background noise from local stationary objects in radar: in both cases the operation of subtraction of signals is using though in different ways. Some experimental results are presented on Figure 5. Standard signal processing has included beam forming, correlation processing of LFM probe signals and threshold detection. Equivalent radius of moving object was about $(-17 \mathrm{~dB})$. Yellow circle is a proposed area of object position. 

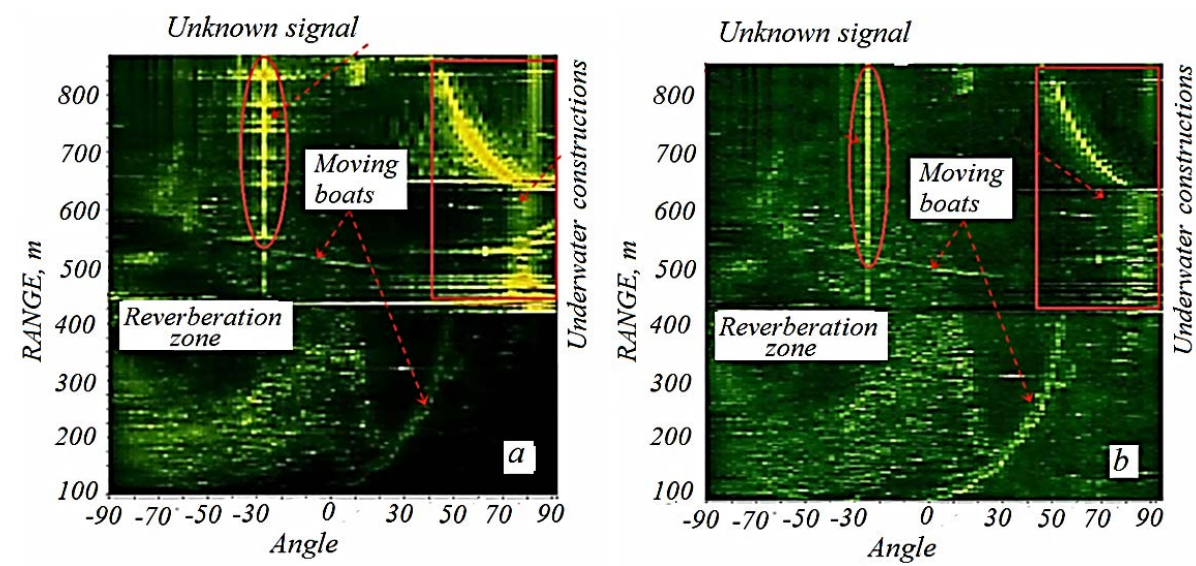

Figure 4. Sonar images of shallow port area. (a) Image in linear mode; (b) Image in clipping mode.
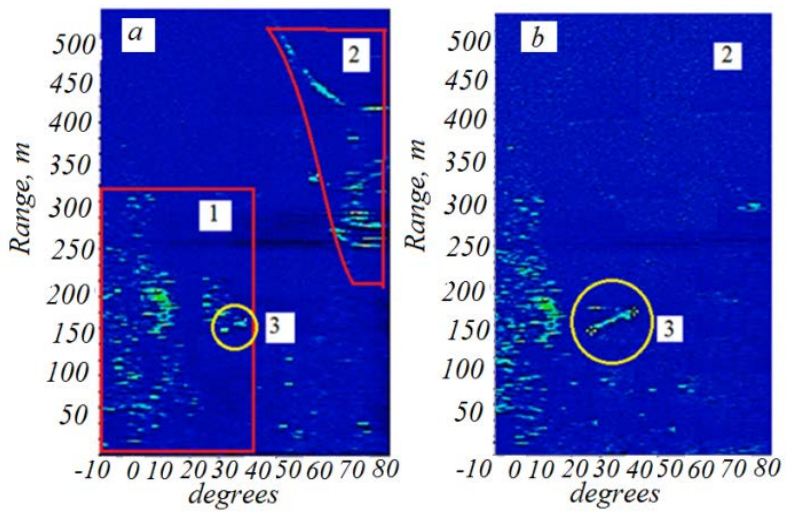

Figure 5. Detection of small object in a shallow sea area. (a). Standard signal processing; (b). Speckle tracking. 1) Reverberation zone; 2) Stationary objects (breakwater); 3) Trajectory of moving object.

One can see that some echo-signals from object are detected but reliable tracking is absent, (zone 3). Left side of image is the reverberation (zone 1) and in zone 2 underwater constructions are clearly seen. The trajectory of moving object after speckle processing is presented on Figure 6(b) (zone 3). Note that suppression of stationary objects was used for horizontal sector $+\left(15^{\circ}-80^{\circ}\right)$ only and echo-signals from zone 2 are deleted completely.

\subsection{Influence of Wakes}

First of all we want to present some experimental results of detection of wakes, generated by high-speed small boats which are constantly observed in seaports. The equivalent scattering radius of these boats with a very low draft is very small, and normally they can be detected only due to the produced turbulent wake. One of the example is presented on Figure 7(a), Figure 7(b). In this experiment the speed of the boat was about 13 knots. Images are in Cartesian coordinates. It is clearly seen that "life" time of this wake is rather big-the wake is clearly detected in 20 minutes, though it expands and weakens.

\subsection{The Influence of Wakes on the Detection}

Next data demonstrate the influence of wake on the detection of small underwater moving target, Figures 8(a)-(c). On the frame Figure 8(a) two objects are detected. Signal processing is a correlation compression of LFM signals. One can see the trajectory of moving boat and small moving object on distance about $450 \mathrm{~m}$. The object was on the depth about 4 meters and distance between object and boat was about 55 meters. The object was reliably detected only because of the turbulent layer has not yet enough time to develop on a big depth and spacing 


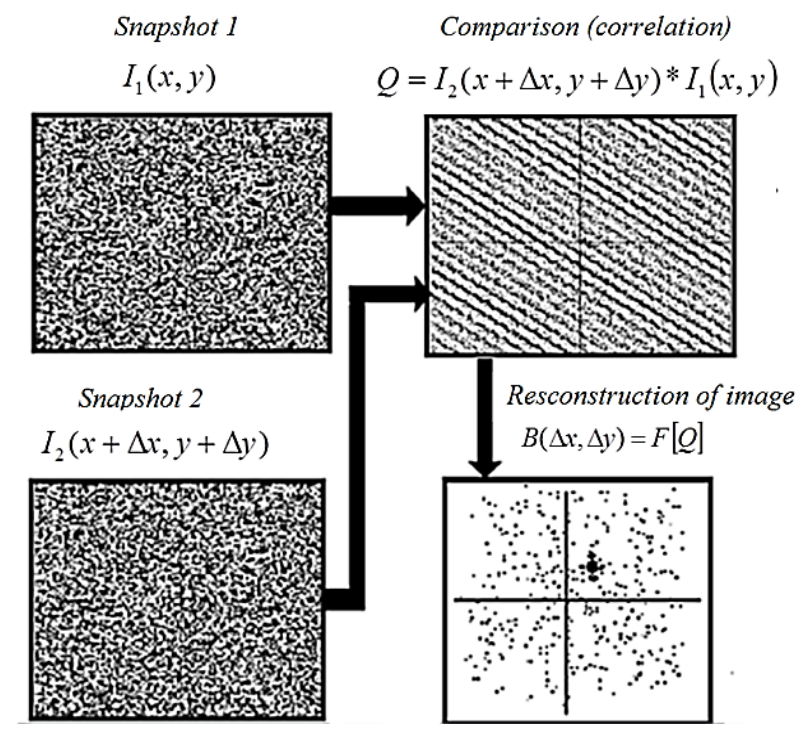

Figure 6. Reconstruction of image of point source from correlation of two speckle patterns.
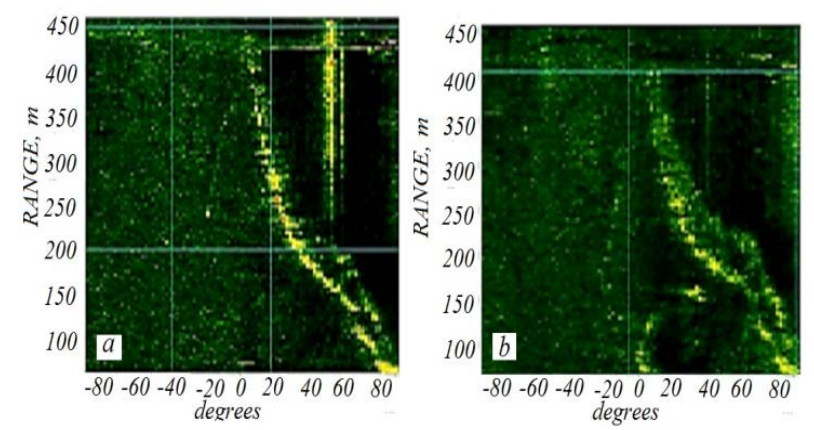

Figure 7. (a) Trajectory of wake from high speed small boat; (b) The same wake after 20 minutes.

between object and boat was rather big. After 16 - 18 minutes a turbulent layer had developed in depth, and the object appeared in its zone, Figure 8(b). Blue marker lines on Figure 8(b) indicate the proposed location of the object which is not detectable. Only after the next 20 minutes the object was detected on the distance about 305 meters, Figure 8(c).

\subsection{The Influence of Strong Noise Generated by Moving Ship}

The next data demonstrate the effectiveness of speckle tracking in a presence of strong reverberation. The average depth of ocean port area was about $12 \mathrm{~m}$ and the depth of receiving array was 4, 5 meters. Standard correlation processing did not give the image of reliable trajectory of moving object, though sometimes it was reliably detectable: red dots on the image Figure 9(a). Correlation processing of clipped signals with speckle tracking practically provided constant detection of the moving object.

\section{Analysis and Discussion}

Several problems hamper the effective detection of small objects moving in shallow areas and especially in port areas. Shallow depth leads to a strong multipath sound propagation that makes use highly directional arrays in both angular planes. Heavy shipping traffic generates several types of interference as additive noise (noise of ships and their navigation acoustic equipment) and multiplicative noise caused by turbulent wakes. Set conditions lead to the fact that such propagation channels can not be regarded as channels with fixed and stationary 

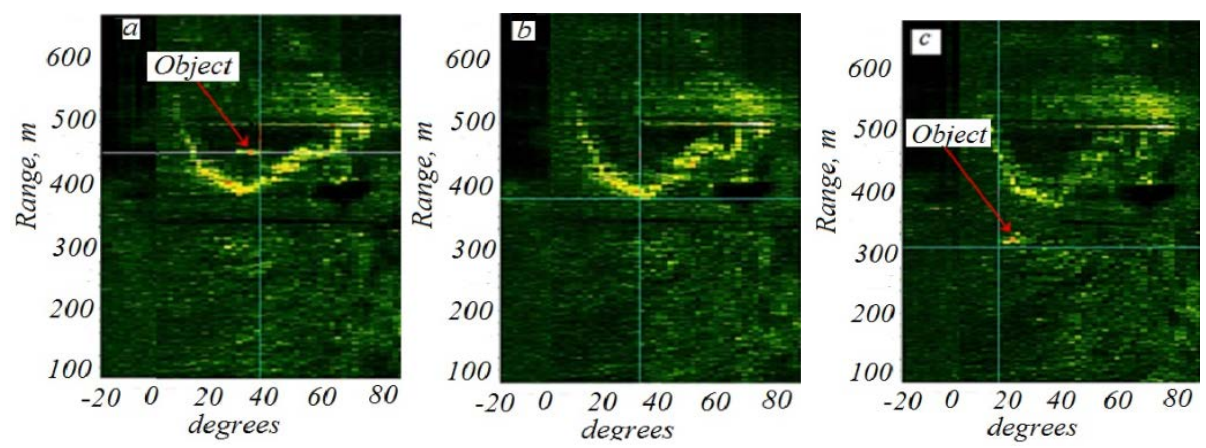

Figure 8. Detectionof object in a wake generated by high speed small boat. Blue markers show the position of object (a) Object is detectable and is behind the wake; (b) The same image after 10 minutes. Object is not detectable; (c) The same image in 15 minutes. Object is detectable. Pay attention on a big life time of wake.
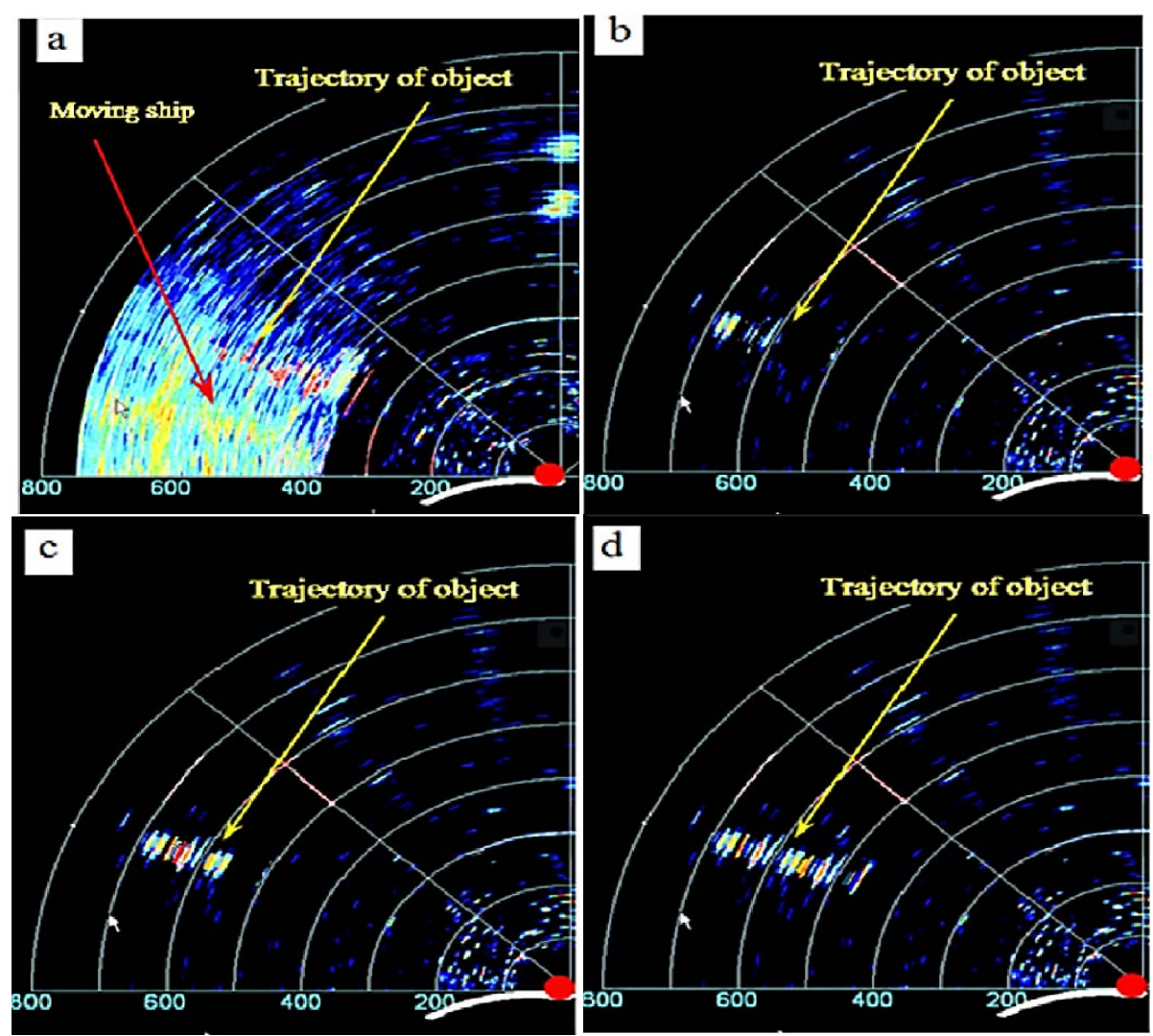

Figure 9. Tracking of moving object in a presence of strong noise generated by moving ship. (a) Standard correlation processing in a linear mode; (b) Correlation procedure of clipped signals and speckle tracking after 10 iterations; (c) Correlation procedure and speckle tracking after 30 iterations; (d) Correlation procedure and speckle tracking after 50 iterations.

parameters. No stationary channel makes use besides of standard procedures the signal processing some additional methods of signal processing, namely adaptive threshold detection in different angle channels, speckle tracking and clipping of input signals because this mode can stabilize false alarm. Despite the fact that the clipped mode at very high signal/noise ratio from strong source reduces the efficiency of the detection of a weak signal, it successfully eliminates noise nonstationarity that it is more important in these conditions. Some experimental data for sea port area are presented on the Figure 10, Figure 11. The dependence of output signal/noise ratio for constant value of threshold is presented on Figure 10 for both modes-linear and clipping mode. First 
of all we can see that moving object with target strength about $(-17 \mathrm{~dB})$ is detected reliably on the distances no more than 300 meters. On bigger distances the object is detectable randomly. Pay attention that real difference between linear mode and clipping mode is small (no more than $2-2.5 \mathrm{~dB}$ ) and this result is in a good correlation with theory.

The introduction of an adaptive threshold with speckle tracking radically changes the situation at bigger distances and the object is confidently detected in both modes, although due to interference nonstationarity clipped mode with speckle tracking works better, since there are no signal gaps, Figure 11. In a linear mode some disappearance of the signals is still observed.

Similar data for other water area are presented in the following Figure 12. The peculiarity of this port area was intensive navigation of vessels with large displacement, multiple signals from echo-sounders and a big number of speed boats.

From Figure 12 it follows that in these conditions standard signal processing even with an adaptive threshold can not provide detection of small object on big distances and in the same time clipping mode with correlation and speckle tracking provide reliable detection on big distances.

\section{Conclusion}

Standard correlation signal processing in unsteady conditions of sound propagation in shallow areas can not provide reliable detection and tracking of underwater small moving objects even with the adaptive threshold in all angular channels. Clipping mode in conjunction with sign correlation and speckle-tracking processing can significantly increase the probability of detection and obtain a stable track of a moving object. Wakes are strongly influence on detection capabilities, but today it is not so clear how to overcome these effects in combined mode "receiving-transmitting". If for some practical applications the wakes are basic and prevalent factor

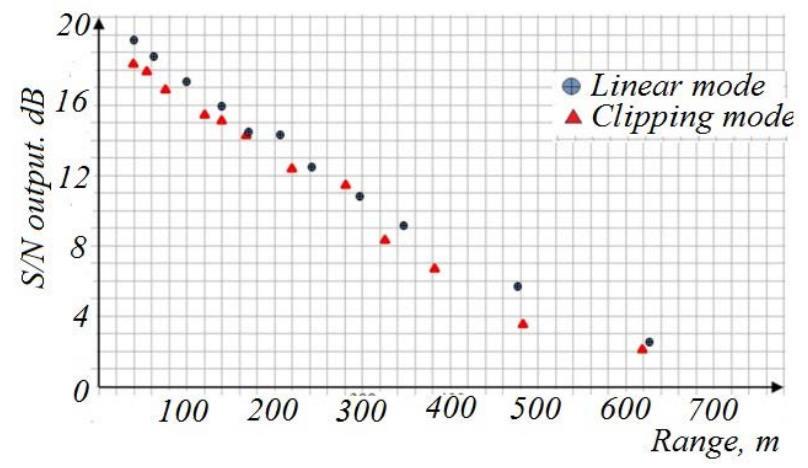

Figure 10. Output signal/noise ratio versus distance for linear mode and clipping mode at constant value of threshold.

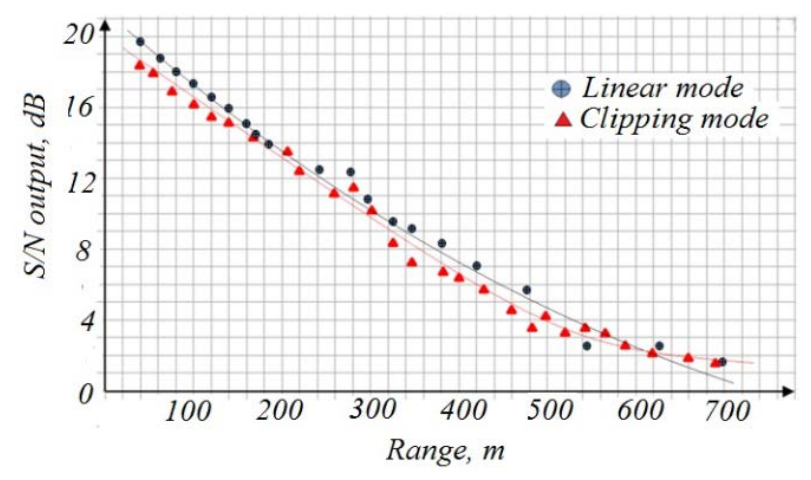

Figure 11. Dependence of output Signal/Noise ratio of range for different modes of signal processing with adaptive threshold. Circles - linear mode, triangles - clipping mode with speckle tracking. 


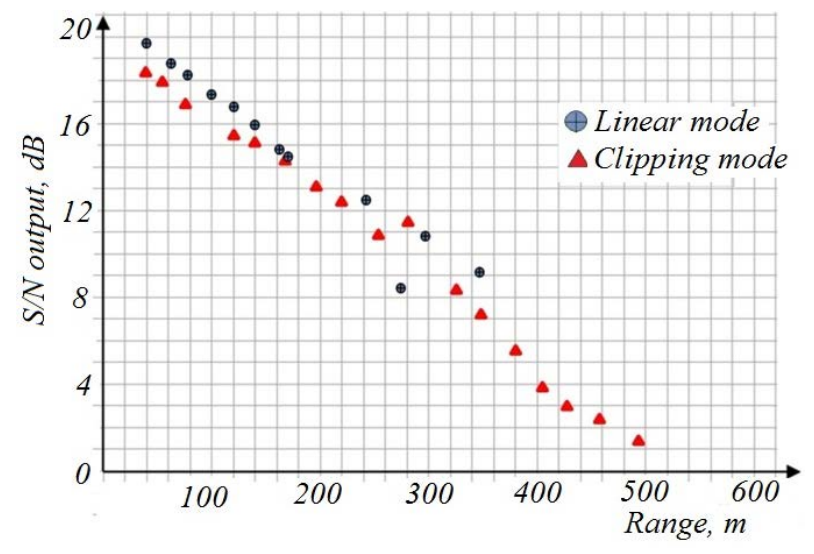

Figure 12. Sea port area 2. Dependence of output signal/noise ratio for different modes of signal processing with adaptive threshold. Circles - linear mode. Triangles - clipping mode with speckle tracking.

preventing detection, it is necessary to use the TRA although from a technical point of view it is more complex for practical realization in port areas.

\section{References}

[1] Zuikova, N.V. and Svet, V.D. (1993) Matched Filed Processing in Waveguides with Sound Velocity Profile. Akusticheskij Zhurnal (Review), 39, 389-403.

[2] Baggeroer, A.B., Kuperman, W.A. and Mikhalevsky, P.N. (1993) An Overview of Matched Field Methods in Ocean Acoustics. IEEE Journal of Oceanic Engineering, 18, 401-424. http://dx.doi.org/10.1109/48.262292

[3] Fink, M. (1992) Time Reversal of Ultrasonic Fields Part 1: Basic Principles. IEEE Transactions on Ultrasonic, Ferroelectrics, and Frequency Control, 39, 555-566. http://dx.doi.org/10.1109/58.156174

[4] Chernyak. Yu. (1962) About Linear Features of System "Broadband Limiter-Filter". Radio technique and Electronics 7, 1073-1-76 (in Russian).

[5] Svet, V.D., Zuikova, N.V. and Kondrat'eva, T.V. (1996) Application of Speckle Interferometry to Problems of Ocean Acoustics. Akusticheskij Zhurnal, 42, 225-231.

[6] Zuikova, N.V., Kondrat'eva, T.V. and Svet, V.D. (1998) Estimating the Angular Movement of a Sound Source in a Multimode Waveguide. Akusticheskij Zhurnal, 44, 220-225.

[7] Zuikova, N.V., Svet, V.D. and Shatskov, Yu.A. (2006) Determination of the Path of a Sound Source Moving in an Inhomogeneous Medium. Akusticheskij Zhurnal, 52, 655-664.

[8] Svet, V.D., Zuikova, N.V. and Kondrat'eva, T.V. (2003) Acoustic Images of Objects Moving under an Inhomogeneous Layer. Akusticheskij Zhurnal, 49, 183-193.

[9] Burch, J.M. and Tokarski, J.M.J. (1968) Production of Multiple Beam Fringes from Photographic Scatterers. Optica Acta, 101-111. 
Scientific Research Publishing (SCIRP) is one of the largest Open Access journal publishers. It is currently publishing more than 200 open access, online, peer-reviewed journals covering a wide range of academic disciplines. SCIRP serves the worldwide academic communities and contributes to the progress and application of science with its publication.

Other selected journals from SCIRP are listed as below. Submit your manuscript to us via either submit@scirp.org or Online Submission Portal.
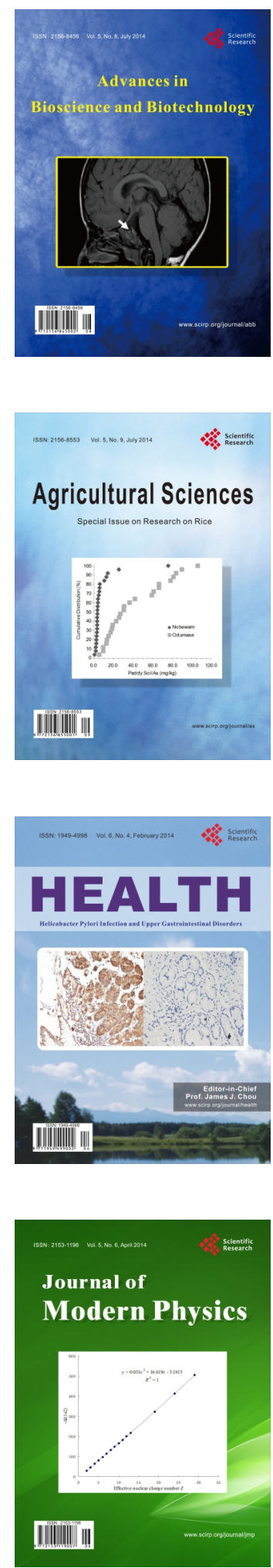
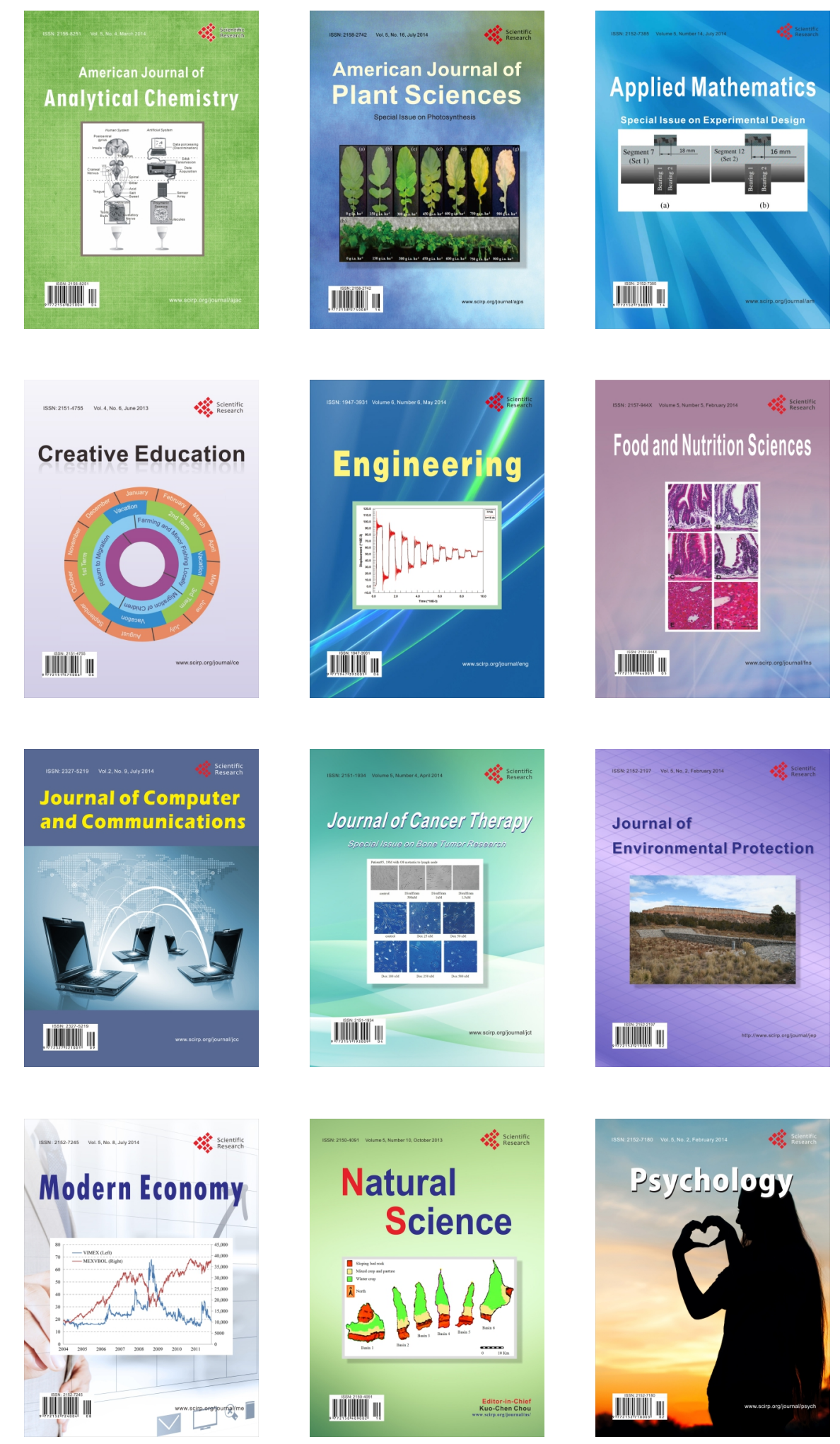